\title{
PREVALÊNCIA DE AGENESIA DENTÁRIA EM PACIENTES SUBMETIDOS A TRATAMENTO ORTODÔNTICO: UM ESTUDO RADIOGRÁFICO
}

\section{PREVALENCE OF DENTAL AGENESIS IN PATIENTS SUBMITTED TO ORTHODONTIC TREATMENT: A RADIOGRAPHIC STUDY}

\author{
Camila Afonso Paravizo (D), Mônica Souza Pinto (D), Roberta Mansur-Caetano* (D), Alcemar \\ Gasparini Netto (D), Maira Tavares de Faria (iD)
}

Centro Universitário de Volta Redonda, Volta Redonda, RJ, Brasil.

*robmansur@hotmail.com

\section{RESUMO}

A anomalia dentária de desenvolvimento mais comum na dentição humana é a agenesia dentária, que pode acontecer nas dentições decídua e permanente. Os dentes mais acometidos pela agenesia são os terceiros molares, pré-molares e incisivos laterais superiores. Comumente está associada a outros tipos de anomalias como a microdontia, ectopias e atraso no desenvolvimento dentário. Portanto, o cirurgião dentista deve se atentar para o desenvolvimento de outras anomalias para que o diagnóstico e a interceptação ortodôntica aconteçam em momento oportuno. Foi realizada uma avaliação das radiografias panorâmicas da documentação de 50 pacientes, selecionados aleatoriamente, com idade entre 6 e 20 anos, de ambos os gêneros, excluindo pacientes sindrômicos, que buscaram tratamento ortodôntico no Curso de Pós-graduação em Ortodontia do Centro Universitário de Volta Redonda. O objetivo desse estudo observacional foi avaliar a presença de agenesia de algum elemento dentário permanente, excluindo os terceiros molares, identificar os dentes mais acometidos, bem como, a associação com outras anomalias dentárias. Avaliando os resultados observou-se que 9 pacientes apresentavam agenesia dentária (18\%), com maior prevalência no gênero feminino $(55,6 \%)$. Os dentes mais acometidos foram os incisivos laterais superiores, seguido dos segundos pré-molares inferiores e segundos pré-molares superiores. A presença de outras anomalias dentárias associadas à agenesia foi identificada em 4 pacientes (44,4\%), com predomínio no gênero feminino (75\%), que foram o atraso no desenvolvimento dentário, forma conóide, ectopia no posicionamento e dilaceração radicular. Concluiu-se que na amostra estudada houve alta prevalência de agenesia dentária, tendo como referência outros estudos com pacientes ortodônticos.

Palavras-chave: Anodontia. Anomalia dentária. Ortodontia.

\section{ABSTRACT}

The most common developmental dental anomaly in human dentition is the dental agenesis which can happen in the deciduous and permanent dentitions. The most affected teeth by the agenesis are the third molars, premolars and upper lateral incisors. It is commonly associated with other types of anomalies such as microdontia, ectopias and delayed dental development. Therefore, the dental surgeon must pay attention to the development of other anomalies so that the diagnosis and orthodontic interception can happen in the appropriate moment. An evaluation of panoramic radiographs and medical records of 50 patients, randomly selected, aged between 6 and 20 years, of both genders, excluding syndromic patients, who sought orthodontic treatment in the Postgraduate Course in Orthodontics at Volta Redonda University Center, was carried out. The objective of this observational study was to evaluate the presence of agenesis of some permanent dental element, excluding the third molars, to identify the most affected teeth, as well as the association with other dental anomalies. Evaluating the results, it was observed that 9 patients had dental agenesis (18\%), with a higher prevalence in females (55.6\%). The most affected teeth were the upper lateral incisors, followed by the lower second premolars and second upper premolars. The presence of other dental anomalies associated with agenesis was identified in 4 patients $(44.4 \%)$, with a predominance in females $(75 \%)$, which were the delayed tooth development, conoid shape, ectopia in positioning and root laceration. It was concluded that in the studied sample there was a high prevalence of dental agenesis, having as reference other studies with orthodontic patients.

Keywords: Anodontia. Tooth abnormalities. Orthodontics. 


\section{INTRODUÇÃO}

A dentição mista é caracterizada pela substituição dos dentes decíduos por permanentes e nesse processo algumas irregularidades odontogênicas podem ocorrer, com diversos graus de severidade, que são chamadas anomalias dentárias de desenvolvimento (SIRIANNI; GONÇALVES, 2019).

A agenesia dentária representa a ausência de formação do elemento dentário, anomalia de desenvolvimento mais comum na dentição humana, com prevalência aproximada de $25 \%$ na população, apresentando como provável fator etiológico, a genética (GARIB; ALENCAR, 2013).

É uma anomalia rara na dentição decídua, entretanto, a ausência de um dente decíduo apresenta um grau de recorrência de 95\% na dentição permanente (SILVA FILHO; GARIB, 2013; NEVILLE et al., 2016).

Os dentes mais frequentemente acometidos por agenesia são os terceiros molares, seguido pelos segundos pré-molares inferiores, logo após, os incisivos laterais superiores e segundos prémolares superiores (GARIB; ALENCAR, 2013; NEVILLE et al., 2016; MARTINS, 2018).

Um mesmo defeito genético pode originar diferentes manifestações fenotípicas, o que explica o fato da agenesia dentária geralmente se associar a outros tipos de anomalias como a microdontia, ectopias e atraso no desenvolvimento dentário, portanto, a detecção precoce de uma anomalia serve de alerta ao cirurgião dentista para a possibilidade do desenvolvimento de outras anomalias, no mesmo paciente ou em familiares, permitindo o diagnóstico e tratamento ortodôntico, quando este se fizer necessário, em momento oportuno (GARIB; ZANELLA; PECK, 2005; GARIB et al., 2010).

O objetivo desse estudo foi avaliar a prevalência de agenesia dentária, os dentes mais acometidos, bem como, a associação com outras anomalias dentárias de desenvolvimento, em pacientes submetidos a tratamento ortodôntico no Centro Universitário de Volta Redonda (UniFOA).

\section{MATERIAL E MÉTODOS}

Trata-se de um estudo observacional aprovado pelo Comitê de Ética em Pesquisa do Centro Universitário de Volta Redonda-RJ, em 9 de abril de 2020, com número CAAE 29868620.4.0000.5237. Para construção do referencial teórico foram analisados trabalhos científicos, principalmente a partir de 2010, indexados nas bases de dados LILACS, MEDLINE e SciELO.

Foi realizada uma avaliação das radiografias panorâmicas e da documentação odontológica de 50 pacientes, selecionados aleatoriamente, que iniciaram tratamento ortodôntico no Curso de Pós-graduação em Ortodontia do UniFOA, no período de novembro de 2019 a março de 2020.

Foram incluídos os exames de pacientes de ambos os gêneros, com idade entre 6 e 20 anos.

Foram excluídos os exames de pacientes sindrômicos e os exames sem qualidade para realizar a devida interpretação.

Foi considerado agenesia a ausência de imagem radiográfica do dente ou de sua cripta alveolar. Os exames nos quais a agenesia dentária foi detectada, foram avaliados a presença de outros tipos de anomalias dentárias de desenvolvimento associadas.

Os terceiros molares foram excluídos desta pesquisa devido à idade dos pacientes que constituíram a amostra, não se podendo afirmar, em alguns casos, se a ausência era congênita ou se o desenvolvimento desses dentes ainda não havia se iniciado.

As imagens foram interpretadas por estudantes do último ano do curso de Odontologia do UniFOA, autoras desse estudo, com a professora orientadora, utilizando-se negatoscópio e lupa, com condições ideais de iluminação. 


\section{RESULTADOS E DISCUSSÃO}

Foi detectado um total de nove pacientes (18\%) com agenesia dentária, excluindo os terceiros molares (Figuras 1 e 2).

Figura 1 - Agenesia dos elementos 12 e 22

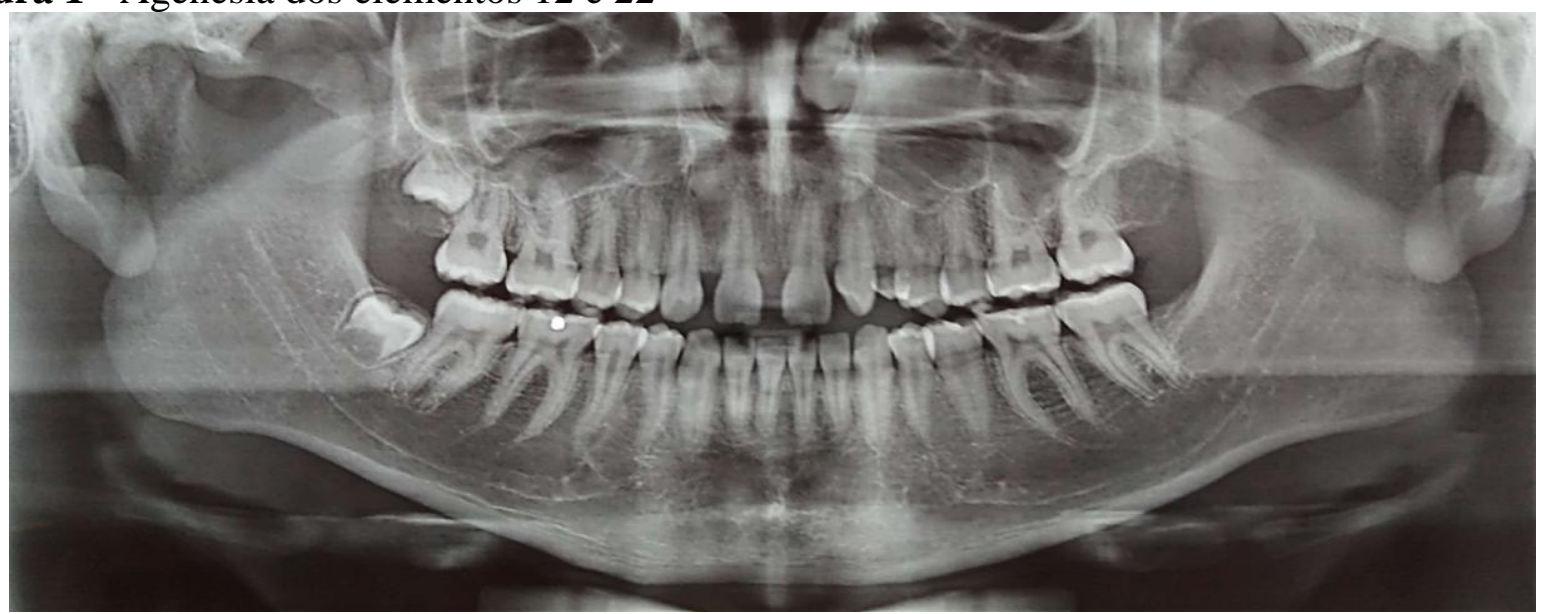

Fonte: os autores.

Figura 2 - Agenesia dos elementos 12 e 22; atraso no desenvolvimento do 35

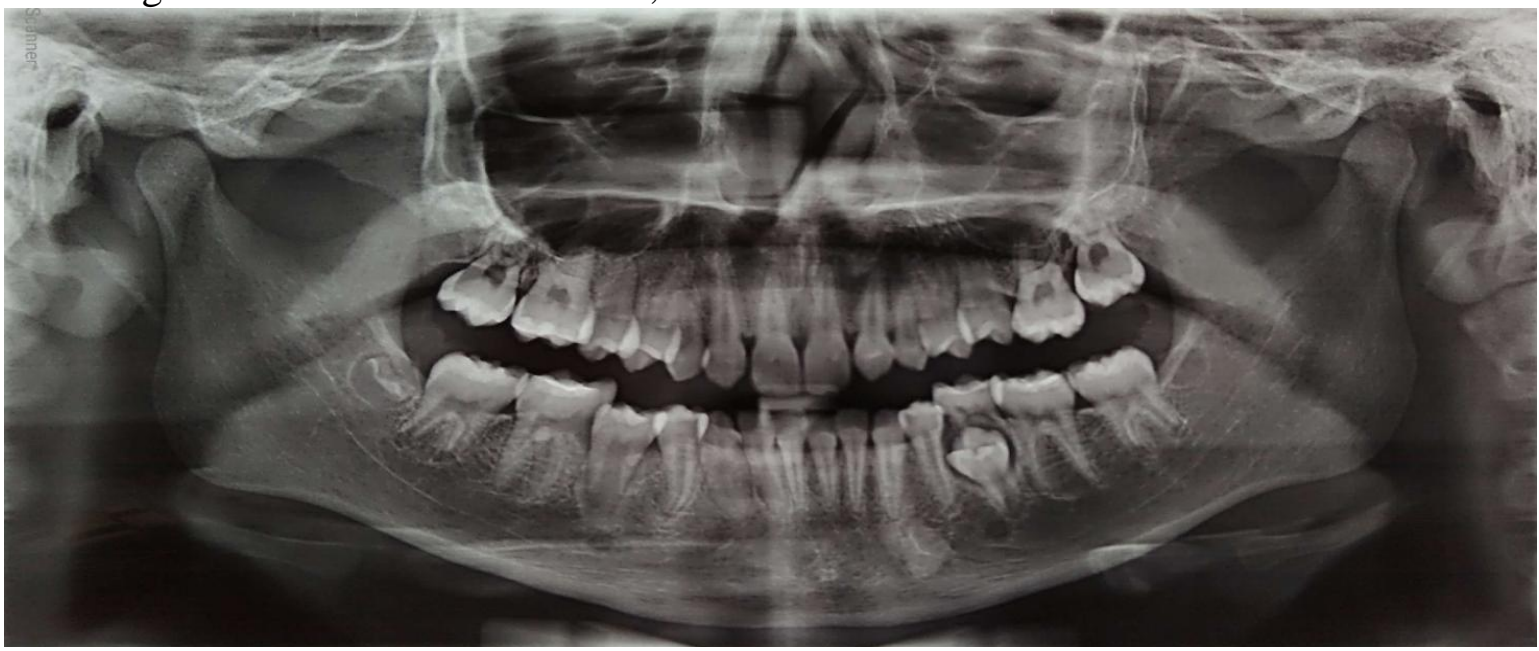

Fonte: os autores.

Em estudos com amostra de pacientes ortodônticos, de faixa etária semelhante e com o mesmo critério de exclusão foram identificados valores inferiores, como o estudo de Pinheiro, Tostes e Pinheiro (2008) com 4,73\%; Torres et al. (2015) com 4,9\% e os estudos de Lotito e Araújo (2017) e Soares (2018), ambos com prevalência de agenesia de 5\%, sendo que nos 4 estudos as amostras eram maiores, respectivamente 1.054,402, 1.129 e 1.902 participantes.

Nos estudos consultados, foram apresentados menores prevalências de agenesia, quando foram avaliados pacientes de maneira geral e não apenas pacientes ortodônticos, como também, quando se excluíam os terceiros molares, como no estudo de França (2011) detectando agenesia em apenas 2,8\% dos pacientes. Por outro lado, os valores eram maiores quando os terceiros molares eram incluídos, como no estudo de Martins Neto et al. (2019) que identificaram 3,9\% de agenesia; estudo de Carvalho e Rodini (2003) 6,8\% e de Arrué (2017) 28,7\%.

Com relação a prevalência da agenesia nos gêneros, no presente estudo foram detectados valores superiores no gênero feminino $(55,6 \%)$, em 5 pacientes, comparado ao gênero masculino $(44,4 \%)$, em 4 pacientes. 
Essa maior prevalência no gênero feminino também foi encontrada na maioria dos estudos (CASTRO; OLIVEIRA; SALES, 2004; FRANÇA, 2011; LOTITO; ARAÚJO, 2017; SOARES, 2018; MARTINS NETO et al., 2019), entretanto, algumas pesquisas relataram não haver diferença significativa entre os gêneros (CARVALHO; RODINI, 2003; TORRES et al., 2015; SIRIANNI; GONÇALVES, 2019).

Dos nove pacientes que apresentavam agenesia dentária no atual estudo, foi detectado ausência de 28 elementos, sendo encontrado oligodontia em dois pacientes (22,2\%), com ausência de sete e nove elementos, e ainda, um paciente com ausência de três elementos, três com ausência de dois elementos e três com ausência de 1 elemento.

Os 28 dentes ausentes foram distribuídos da seguinte forma: 11 incisivos laterais superiores $(39,2 \%), 6$ segundos pré-molares inferiores $(21,4 \%), 4$ segundos pré-molares superiores $(14,2 \%), 2$ primeiros pré-molares superiores $(7,1 \%)$ e 2 incisivos centrais inferiores $(7,1 \%)$, além de $1^{\circ}$ e $2^{\circ}$ molar superior e $1^{\circ}$ molar inferior.

Os dentes mais acometidos de agenesia foram os incisivos laterais superiores, como nos estudos de Torres et al. (2015) e Arrué (2017), e como no estudo de Arrué (2017) o incisivo lateral com maior prevalência foi o elemento 12 .

Entretanto, na literatura pesquisada, também excluindo os terceiros molares, identificou-se maior prevalência da agenesia dos segundos pré-molares inferiores (CARVALHO; RODINI, 2003; GARIB; ALENCAR, 2013; NEVILLE et al., 2016; LOTITO; ARAÚJO, 2017; MARTINS, 2018; SOARES, 2018).

De acordo com Garib et al. (2010) existe uma inter-relação genética no desenvolvimento de algumas anomalias dentárias, portanto, os profissionais devem estar alertas para identificar pacientes com padrão de anomalias dentárias associadas, que se expressam com diferentes graus de severidade.

Avaliando a presença da associação de anomalias de desenvolvimento, dos nove pacientes com agenesias dentárias, quatro $(44,4 \%)$ apresentavam outra anomalia dentária associada, que foram, atraso no desenvolvimento dentário do elemento 35, forma conóide do elemento 22, ectopia no posicionamento do elemento 23, ectopia no posicionamento do elemento 11 e dilaceração radicular dos elementos 37 e 47 . Dos 4 pacientes com outra anomalia dentária associada, 3 (75\%) eram do gênero feminino (Figura 3).

Figura 3 - Associação a outras anomalias dentárias

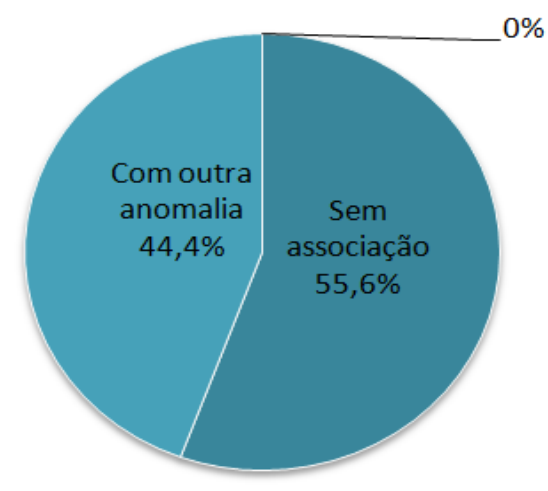

Fonte: os autores.

Vários estudos também avaliaram essa associação, como resultado do mesmo defeito genético:

Peck, Peck e Kataja (1998) analisaram 60 pacientes, idade média 9 a 12 anos, com transposição do incisivo lateral e canino inferiores, sendo detectado uma associação estatisticamente significativa com agenesia dentária $(\mathrm{p}<0,01)$ e forma conóide $(\mathrm{p}<0,001)$ nos incisivos laterais superiores. 
Garib, Peck e Gomes (2009) avaliaram 203 pacientes, de 8 a 22 anos de idade, com agenesia de pelo menos um segundo pré-molar e comparando com outros estudos, concluíram que esses pacientes apresentavam maior prevalência de outras anomalias dentárias, tais como microdontia de incisivos laterais superiores, infra-oclusão de molares decíduos, distoangulação de segundo prémolar inferior e posição ectópica do canino superior para palatina.

Soares (2018) identificou em $80 \%$ dos casos de agenesia, associação com retenção prolongada do dente decíduo, erupção ectópica do canino, dente com forma conóide, microdontia e anquilose dentoalveolar em dentes decíduos.

As anomalias dentárias encontradas associadas à agenesia, identificadas ao final deste estudo, com exceção da dilaceração radicular, também foram mencionadas nos estudos citados. Sendo que, a maior prevalência desse padrão de associação de anomalias aconteceu no gênero feminino, assim como citado por Garib e Alencar (2013).

\section{CONCLUSÃO}

$\mathrm{Na}$ amostra avaliada observou-se que $18 \%$ dos pacientes apresentavam agenesia dentária, com maior prevalência no gênero feminino $(55,6 \%)$. Os dentes mais acometidos foram os incisivos laterais superiores $(39,2 \%)$, seguido dos segundos pré-molares inferiores $(21,4 \%)$ e segundos prémolares superiores $(14,2 \%)$. A presença de outras anomalias dentárias associadas à agenesia foi identificada em 44,4\% dos pacientes, com predomínio no gênero feminino, que foram o atraso no desenvolvimento dentário, forma conóide, ectopia no posicionamento e dilaceração radicular.

Concluiu-se que na amostra estudada houve alta prevalência de agenesia dentária, tendo como referência outros estudos com pacientes ortodônticos.

\section{REFERÊNCIAS}

ARRUÉ, T. A. Prevalência de Anomalias Dentárias em Pacientes Pediátricos. 2017. 36p. Trabalho de Conclusão de Curso (Graduação em Odontologia) - Universidade Federal do Rio Grande do Sul. Faculdade de Odontologia, Porto Alegre, 2017.

CARVALHO, M. C.; RODINI, E. S. O. Estudo da prevalência de alterações dentárias hipoplasiantes em uma amostra da população de Bauru. Salusvita, v. 22, n. 2, p. 191-199, 2003.

CASTRO, J. F. L.; OLIVEIRA, S. B.; SALES, R. D. Prevalência das anomalias dentárias em pacientes submetidos a Tratamento ortodôntico. Revistas de Ortodontia Dental Press, v. 9, n. 5, p. 79-84, 2004.

FRANÇA, M. M. C. Incidências das Agenesias de dentes permanentes na população de Uberlândia-MG. 2011. 78p. Dissertação (Mestrado em Clínica Odontológica Integrada) Universidade Federal de Uberlândia, Uberlândia, 2011.

GARIB, D. G.; ALENCAR, B. M. Padrão de anomalias dentárias associadas. In: SILVA FILHO, O. G.; GARIB, D. G.; LARA, T. S. Ortodontia Interceptiva: Protocolo de tratamento em duas fases. São Paulo: Artes Médicas, 2013.

GARIB, D. G. et al. Anomalias dentárias associadas: o ortodontista decodificando a genética que rege os distúrbios de desenvolvimento dentário. Revistas de Ortodontia Dental Press, v. 15, n. 2, p. 138-157, 2010.

GARIB, D. G.; PECK, S.; GOMES, S. C. Increased Occurrence of Dental Anomalies Associated with Second-Premolar Agenesis. The Angle Orthodontist, v. 79, n. 3, p. 436-441, 2009. 
GARIB, D. G.; ZANELLA, N. L. M.; PECK, S. Associated dental anomalies: case report. Journal of Applied Oral Science, v. 13, n. 4, p. 431-436, 2005.

LOTITO, M. C. F.; ARAÚJO, M. T. S. Prevalência de agenesia dentária em pacientes tratados na clínica de ortodontia da faculdade de Odontologia da Universidade Federal do Rio de Janeiro. Revista Ortodontia Gaúcha, v. 22, n. 1, 2017.

MARTINS, R. M. N. Agenesia de segundos pré-molares - Associação com outras agenesias. 2018. 39p. Dissertação (Mestrado Integrado em Medicina Dentária) - Universidade de Lisboa, Lisboa, 2018.

MARTINS NETO, R. S. et al. Prevalência de anomalias dentárias em radiografias panorâmicas. Archives of Health Investigation, v. 8, n. 2, p. 68-73, 2019.

NEVILLE, B. W. et al. Patologia Oral e Maxilofacial. 4. ed., Rio de Janeiro: Elsevier, 2016.

PECK, S.; PECK, L.; KATAJA, M. Mandibular lateral incisor- canine transposition concomitant dental anomalies, and genetic control. The Angle Orthodontist, v.68, n.5, p.455-466, 1998.

PINHEIRO, C. C.; TOSTES, M. A.; PINHEIRO, A. R. Prevalência de Anomalia Dentária de Número em Pacientes Prevalência de Anomalia Dentária de Número em Pacientes Submetidos a Tratamento Ortodôntico: um Estudo Radiográfico. Pesquisa Brasileira em Odontopediatria e Clínica Integrada, v. 8, n. 1, p. 47-50, 2008.

TORRES, P. F. et al. Anomalias Dentárias de Número em Paciente Ortodônticos. Revista de Odontologia da UNESP, v. 44, n. 5, p. 280-284, 2015.

SILVA FILHO, O. G.; GARIB, D. G. Dentadura decídua: Aspectos clínicos e radiográficos. In: SILVA FILHO, O. G.; GARIB, D. G.; LARA, T. S. Ortodontia Interceptiva: Protocolo de tratamento em duas fases. São Paulo: Artes Médicas, 2013.

SIRIANNI, L. O.; GONÇALVES, A. L. C. A. Avaliação da prevalência de agenesia de segundos pré-molares dos pacientes do curso de Odontologia do Centro Universitário da Serra Gaúcha. Journal of Oral Investigations, v. 8, n. 2, p. 7-18, 2019.

SOARES, E. C. B. Prevalência de Hipodontia em Pacientes Ortodônticos Tratados em Belo Horizonte, Brasil. 2018. 23p. Monografia (Especialização em Ortodontia) - Universidade Federal de Minas Gerais, Belo Horizonte, 2018. 